\title{
A multilevel analysis of craniofacial growth in subjects with untreated Class III malocclusion
}

\author{
Valentina Rutili $^{1}$ ｜ Michele Nieri ${ }^{1}$ ｜ Veronica Giuntini ${ }^{1}$ ｜ James A. McNamara Jr ${ }^{2,3,4,5}$ | \\ Lorenzo Franchi ${ }^{1,2}$ iD
}

\author{
${ }^{1}$ Section of Dentistry (Orthodontics), \\ Department of Experimental and Clinical \\ Medicine, The University of Florence, Italy \\ ${ }^{2}$ Department of Orthodontics and Pediatric \\ Dentistry, School of Dentistry, The \\ University of Michigan, Ann Arbor, Michigan \\ ${ }^{3}$ Emeritus of Cell and Developmental \\ Biology, School of Medicine, The University \\ of Michigan, Ann Arbor, Michigan \\ ${ }^{4}$ Center for Human Growth and \\ Development, The University of Michigan, \\ Ann Arbor, Michigan \\ ${ }^{5}$ Private Practice of Orthodontics, Ann \\ Arbor, Michigan \\ Correspondence \\ Lorenzo Franchi, Section of Dentistry \\ (Orthodontics), Department of Experimental \\ and Clinical Medicine, Università degli Studi \\ di Firenze, Firenze, Italy. \\ Email: lorenzo.franchi@unifi.it
}

\begin{abstract}
Objective: To analyse the craniofacial growth of a long-term semi-longitudinal sample of Caucasian subjects with untreated Class III malocclusion.

Setting and sample population: A total of 144 Caucasian subjects (of North American and Italian origin) with untreated Class III malocclusion.

Materials and methods: Subjects aged 2 years and 9 months up to 21 years and 7 months were selected. A multilevel model was used to calculate growth curves for ten variables for both each individual subject and for the whole sample.

Results: There was a statistically significant increase for total mandibular length (CoGn. T2-T1 $=8.4 \mathrm{~mm}$ ), midfacial length $($ Co-A. T2-T1 $=3.4 \mathrm{~mm}$ ) and lower anterior facial height (ANS-Me. T2-T1 $=3.8 \mathrm{~mm}$ ). The multilevel analysis showed two points of acceleration of growth (about 3-5 years of age and 11-15 years of age) for seven out of ten variables. For $\mathrm{Co}-\mathrm{Gn}$ and $\mathrm{Co}-\mathrm{A}$ variables, males presented points of maximum growth delayed by 1 year in comparison with females, with a greater duration (1 year longer) and a greater total growth of about $5 \mathrm{~mm}$. Active mandibular growth continued for a long time after the pubertal spurt: increases in mandibular length ended at about 17 years of age in females and at 21 years and 7 months in males.

Conclusions: Untreated Class III malocclusion showed a specific growth curve, especially for the mandible, whose excesses added up over time. In males, the amounts of mandibular and midfacial growth during the whole observation time were greater and lasted longer than in females.
\end{abstract}

\section{KEYWORDS}

caucasian, cephalometrics, Class III, growth, malocclusion

\section{INTRODUCTION}

Although in recent years interest concerning treatment of Class III malocclusion has intensified, ${ }^{1-3}$ studies on craniofacial growth of untreated Class III subjects continue to be scarce. ${ }^{4-8}$ To date, investigators have found that Class III malocclusion is the dentoskeletal disharmony with the lowest prevalence in the world; a global range varies from $0 \%$ to $26.7 \% .^{9}$ The prevalence of this condition depends on ethnicity and geographic region. In Caucasian populations, the prevalence is relatively low (from $1.9 \%$ to $12.2 \%$ ). ${ }^{10-13}$ The highest prevalence is recorded in Southeast Asian populations (in China and in Malaysia, the prevalence is between $12.6 \%$ and $26.7 \%$ ). ${ }^{14-16}$

The complexity of this malocclusion is reflected in its etiopathogenesis. Class III malocclusion is the result of both genetic and environmental factors. The former has a strong unfavourable influence on the course of craniofacial development; thus far, multiple models 
of inheritance have been hypothesized. The autosomal-dominant model with incomplete penetrance and the polygenic inheritance model are two of the most probable explanations. ${ }^{17,18}$

The growth pattern observed in Class III malocclusion is complex. Cross-sectional studies have concluded that its typical craniofacial characteristics already are established at an early age. ${ }^{19,20}$ Class III malocclusion is not susceptible to spontaneous improvement during growth. On the contrary, this condition tends to worsen over time, especially in males ${ }^{4,21-23}$ with excessive mandibular growth. Growth continues to worsen slightly even after the pubertal phase. ${ }^{4,23}$

Differences have been found in the craniofacial growth of subjects with untreated Class III malocclusion compared to Class I subjects. Cross-sectional studies have shown that the pubertal growth spurt lasts longer in subjects with untreated Class III malocclusion compared to that of subjects with Class I malocclusion (on average 5 months longer). ${ }^{24}$ Excessive mandibular growth, the absence of catch-up growth of the maxilla and the vertical direction of facial growth appear to be unfavourable aspects in Class III malocclusion. ${ }^{4,6}$ Moreover, the pubertal growth spurt seems to be delayed for a few years compared with individuals with normal occlusion. $^{23}$

Longitudinal studies available on this topic ${ }^{4-6}$ have confirmed only a few of these aspects. Furthermore, there is a shortage of longitudinal studies with untreated Class III subjects followed until the end of their skeletal growth. Many studies regarding craniofacial growth have outlined polynomial multilevel models. ${ }^{25-28}$ The curvilinear multilevel model can estimate the extent of acceleration (when its regression coefficient is positive) or deceleration (when its regression coefficient is negative). ${ }^{27}$

Only one study regarding craniofacial growth of untreated Class III malocclusion was reported performing a multilevel statistical analysis using a linear model. ${ }^{6}$ Linear multilevel models can show growth velocity but cannot consider variations in the subjects and their oscillations during growth. ${ }^{25,27}$ Thus, the present study is aimed to describe craniofacial growth in a group of untreated Caucasian subjects (of Italian and North American origin) with Class III malocclusion using cephalometric data elaborated with a curvilinear multilevel model including chronological age up to the fifth order.

\section{2 | MATERIALS AND METHODS}

This study was exempted from review by the Medical School Institutional Review Board of the University of Michigan (HUM00160285).

This article is structured according to STROBE guidelines for longitudinal studies. ${ }^{29}$ The sample consisted of semi-longitudinal cephalometric data of Caucasian subjects with untreated Class III malocclusion. It was derived in part from a database of untreated Class III subjects ( 41 subjects: 17 males and 24 females) described by Levin et $\mathrm{al}^{30}$ and in part from a database reported by Zionic Alexander et al ${ }^{5}$ (104 subjects: 48 males, 56 females), for a total of 145 subjects. These patients were left untreated because they refused treatment or because their records were derived from historical samples taken from Growth Center Studies conducted in the USA and Canada.

Subjects originally were selected by orthodontists from the United States and Canada in their private practices. Other sources included university-affiliated orthodontic clinics, Growth Center Studies (including the Bolton-Brush Growth Study, the Burlington Growth Center, the University of Michigan Elementary and Secondary School Growth Study and the Denver Child Growth Study), the Orthodontic Clinic of the University of Michigan and the Orthodontic Clinic of the University of Florence, Italy. 5,30

For each patient, a series of longitudinal lateral cephalograms were available. ${ }^{5,30}$ Each subject had at least two cephalometric records related to two different ages, with the films taken at least one year apart. The distribution of the sample according to the number of cephalometric films for each subject is displayed in Table S1. Cephalometric magnification varied originally from $0 \%$ (life size) to $12.9 \%$, and it was then standardized to $0 \%$.

Patients with pseudo Class III anterior crossbite were excluded for two reasons: first, in contemporary Class III subjects who refused treatment, the orthodontist reported a functional deviation; second, an increase in the linear distance between the second vertebral body and the posterior border of the mandibular ramus during tooth intercuspation was noted for subjects derived from Growth Center Studies.

The sample that met the inclusion criteria (Table 1) consisted of 145 Caucasian patients with untreated Class III malocclusion. Of these subjects, 1 patient showed cephalometric data typed incorrectly; this subject was eliminated. The final number of subjects was 144 (65 males and 79 females). Patients who derived from Growth Center Studies (Growth Study Group) were 45 out of 144; patients who refused Class III treatment (Non-Growth Study Group) were 99 out of 144.

For each subject, a lateral cephalometric record at T1 (time of the patient's first observation: on average $10.0 \pm 3.7$ years) was available, and one at T2 (patient's last observation: on average

\section{TABLE 1 Sample inclusion criteria}

1. European or American ancestry (Caucasian ethnicity)

2. No orthodontic or orthopaedic treatment before the first cephalometric record, or between the records, has been performed

3. Initial diagnosis (T1) of:

a. anterior crossbite (excluding pseudo-crossbite)

b. edge-to-edge incisive relationship, concomitant with one of the skeletal Class III criteria

c. accentuated mesial step relationship of the deciduous second molars

d. mesial Class III relationship of the first permanent molar of at least half cusp

4. Class III skeletal relationship having one or both:

a. a negative Wits appraisal greater than $-2.0 \mathrm{~mm}$

b. an ANB angle less than $0^{\circ}$

5. No congenitally missing or extracted teeth

6. No craniofacial syndromes

7. Not less than 9 mo and not more than 30 mo between consecutive cephalometric films 
至

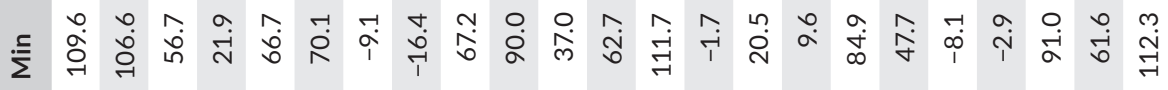

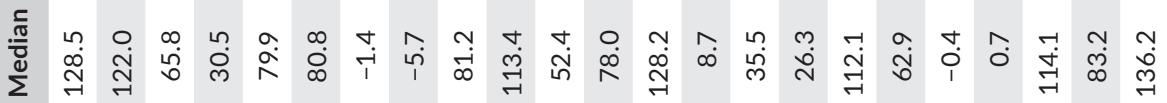

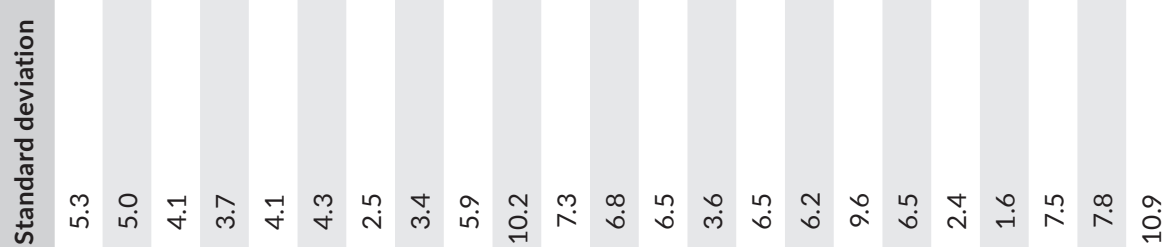

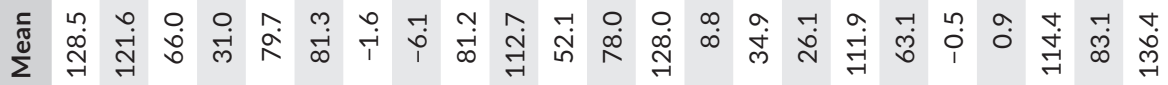

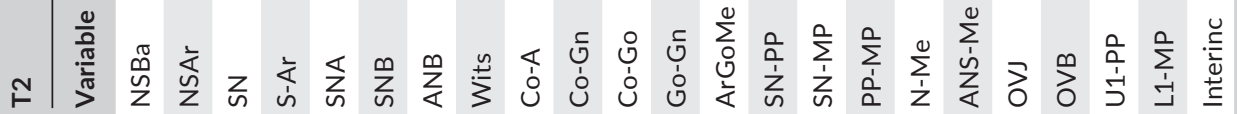

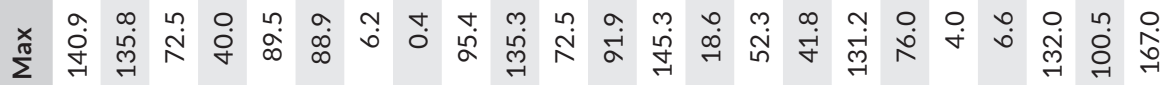

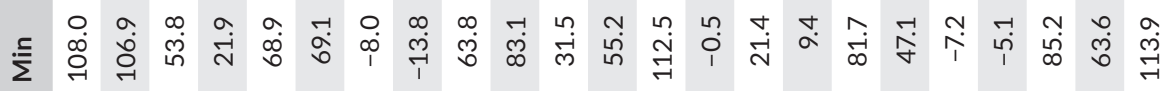

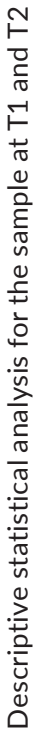

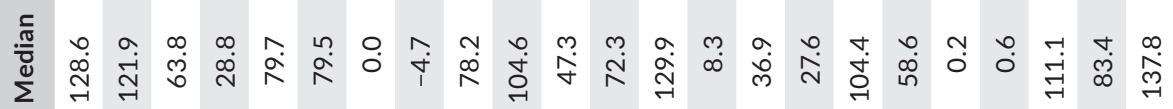

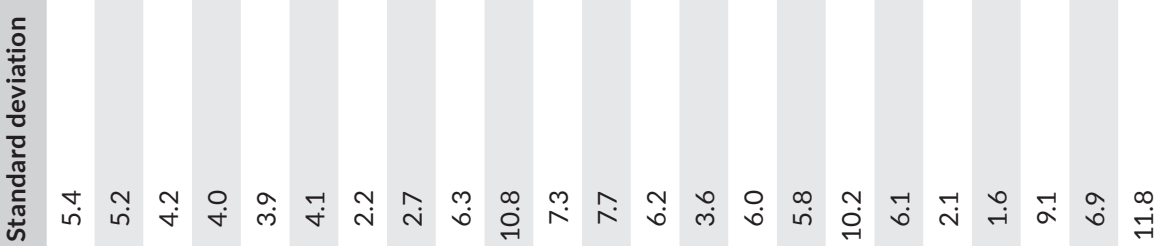

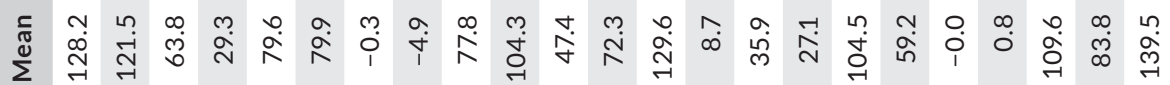

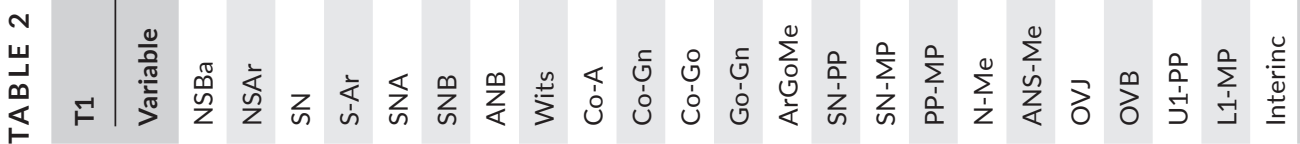


$13.8 \pm 2.7$ years) (Table S2). Patients with observations (cephalometric data) occurring during the post-adolescent age, that is from 18 years of age up to 21 years and 7 months, numbered 16 ( 7 males and 9 females).

\section{1 | Statistical analysis}

The method error for cephalometric measurements is described in Levin et $\mathrm{al}^{30}$ and in Zionic Alexander et al. ${ }^{5}$

Both descriptive and inferential statistical analyses were performed. For the descriptive analysis, 23 cephalometric variables were examined (11 linear and 12 angular) at T1 and at T2, using a statistical software $\left(\mathrm{JMP}^{\circledR}\right.$, version 13.0). Descriptive statistics for the T2-T1 changes for three variables also was calculated for total mandibular length (Co-Gn), midfacial length (Co-A) and lower anterior facial height (ANS-Me). Descriptive statistics and statistical comparisons were performed also for the Growth Study Group vs Non-Growth Study Group at baseline.

An inferential analysis then was performed using a multilevel model at two levels (patient and number of lateral cephalograms). A study of craniofacial growth over time often is performed with longitudinal data across childhood and adolescence. ${ }^{27}$ Analysis of longitudinal data usually requires advanced statistical methods that vary according to properties of the data, such as the number of repeated measurements of the subjects or the shape of the growth curves.

To characterize some features in the process of growth changes, a classical regression analysis can be used. ${ }^{31}$ Regression analysis assumes that the observations are independent, but when multiple observations are collected from the same subjects, those observations are correlated. In particular, in this study a multilevel modelling (also known as hierarchical linear modelling) was conducted to take this correlation into account for analysing longitudinal data.

The advantage of multilevel analysis is that it can show how average growth and individual growth can be characterized. ${ }^{32,33}$ Moreover, it uses polynomials that can define growth curves in many shapes; therefore, multilevel analysis is versatile. ${ }^{32}$ Multilevel analysis also does not require complete longitudinal data; in fact, it can manage missing terms easily. The software used in this study was a multilevel modelling software $\left(\mathrm{MLwiN}^{\circledR}\right.$, version 2.26, University of Bristol, Bristol, UK)

Multilevel analysis examined 10 cephalometric variables (3 linear and 7 angular), that is Co-Gn, Co-A, ANS-Me, NSBa, SNA, SNB, ANB, ArGoMe, SN-MP and PP-MP to evaluate growth variations of these variables over time (including each cephalometric record available) for the whole sample considered. Males and females were analysed separately.

Multilevel models used 'Age' up to the fifth order as an explanatory variable. 'Age ${ }^{5}$ is 'Age' raised to the fifth power. Since growth of the variables does not seem to be linear in the subjects, we have included the term 'Age' up to the fifth order (ie the quintic term of age) in years. A multilevel analysis up to the first term of age would have involved the creation of a linear multilevel graph; this method could not have allowed the highlighting of variations in the craniofacial growth of the variables. ${ }^{31}$ The higher the age orders are, the more accurate is the approximation of the growth curve to the individual observations collected for each patient. Missing values, therefore, can be interpolated with a polynomial method.

The other explanatory variables were 'Gender' and the 'Age $x$ Gender' interaction. The various terms related to 'Age' were left in the model only if significant starting from the polynomial with higher degree. Even the 'Age $x$ Gender' interaction was left in the model only if significant. In any case, the simplest model had to present the variables 'Age' of order 1 and 'Gender'. The level of significance was set at 0.05. The random effects consisted of the 'Intercept' (constant) and the variable 'Age' of order 1 (the latter only if significant).

\section{3 | RESULTS}

Descriptive statistics for the 23 cephalometric variables at T1 and at $\mathrm{T} 2$ are reported in Table 2. Regarding T2-T1 changes for the Co-Gn, Co-A and ANS-Me variables, a significant increase in time interval (Table 3) was found. Midfacial length showed a statistically significant T2-T1 mean increase (3.4 mm. 95\% confidence interval [Cl]: 2.8$3.9 \mathrm{~mm}$ ). This increment, however, was about one third compared to that of total mandibular length $(8.4 \mathrm{~mm}$. Cl: 7.3- $9.5 \mathrm{~mm})$. Moreover, there was a significant increase in the vertical measure ANS-Me in the time interval (3.8 mm. Cl: 3.2- $4.6 \mathrm{~mm}$ ).

Descriptive statistics and statistical comparisons of Growth Study Group vs Non-Growth Study Group at baseline were reported in Table S3. No statistically significant differences were found between the 2 groups with the exception of the angle ANB. The difference in the ANB angle was -1.4 degrees ( $95 \%$ confidence interval from -2.1 degrees to -0.6 degrees)

Growth curves of each of the 144 patients were constructed for the Co-Gn, Co-A and ANS-Me variables at the different ages available. Because this study did not calculate the growth difference of each variable for each age interval considered, the 'spurt' of the growth curve corresponded to a steeper variation of the inclination of the multilevel curve.

TABLE 3 T2-T1 difference of the Co-Gn, Co-A and ANS-Me cephalometric variables

\begin{tabular}{|llcccc}
\hline T2-T1 & Mean & $\begin{array}{c}\text { Standard } \\
\text { deviation }\end{array}$ & Median & Min & Max \\
\hline Co-Gn & 8.4 & 6.6 & 6.2 & 0.3 & 30.7 \\
& Cl 95\% [7.3; 9.5] & & & & \\
\hline Co-A & 3.4 & 3.3 & 2.0 & -1.1 & 16.1 \\
& $\mathrm{Cl} 95 \%[2.8 ; 3.9]$ & & & & \\
\hline ANS-Me & 3.8 & 3.7 & 2.8 & -2.1 & 15.6 \\
& $\mathrm{Cl} 95 \%[3.2 ; 4.6]$ & & & & \\
\hline
\end{tabular}


Multilevel models for ten cephalometric variables are reported in Figures 1 and 2; graphs for multilevel models are presented in Table 4. Longitudinal records by age-class are displayed in Table S4.

The growth trends for total mandibular length (Co-Gn) and midfacial length (Co-A) (Figure 1) for the whole sample were curvilinear, with two growth spikes observed at 3-5 years and $11-15$ years. The spike at 3-5 years should be interpreted with caution due to the limited number of subjects for this age range.
For both variables, there was significant interaction between age and gender; in fact, females showed spurts occurring slightly before males. The growth spurts ended about 1 year earlier in females, and total growth was about $5 \mathrm{~mm}$ smaller compared to males. Total mandibular length growth ceased around 17 years in females. In males, a modest continuation of growth of total mandibular length (Co-Gn) until the end of the observation time (21 years and 7 months) was observed (Figure 1). It should be stressed that
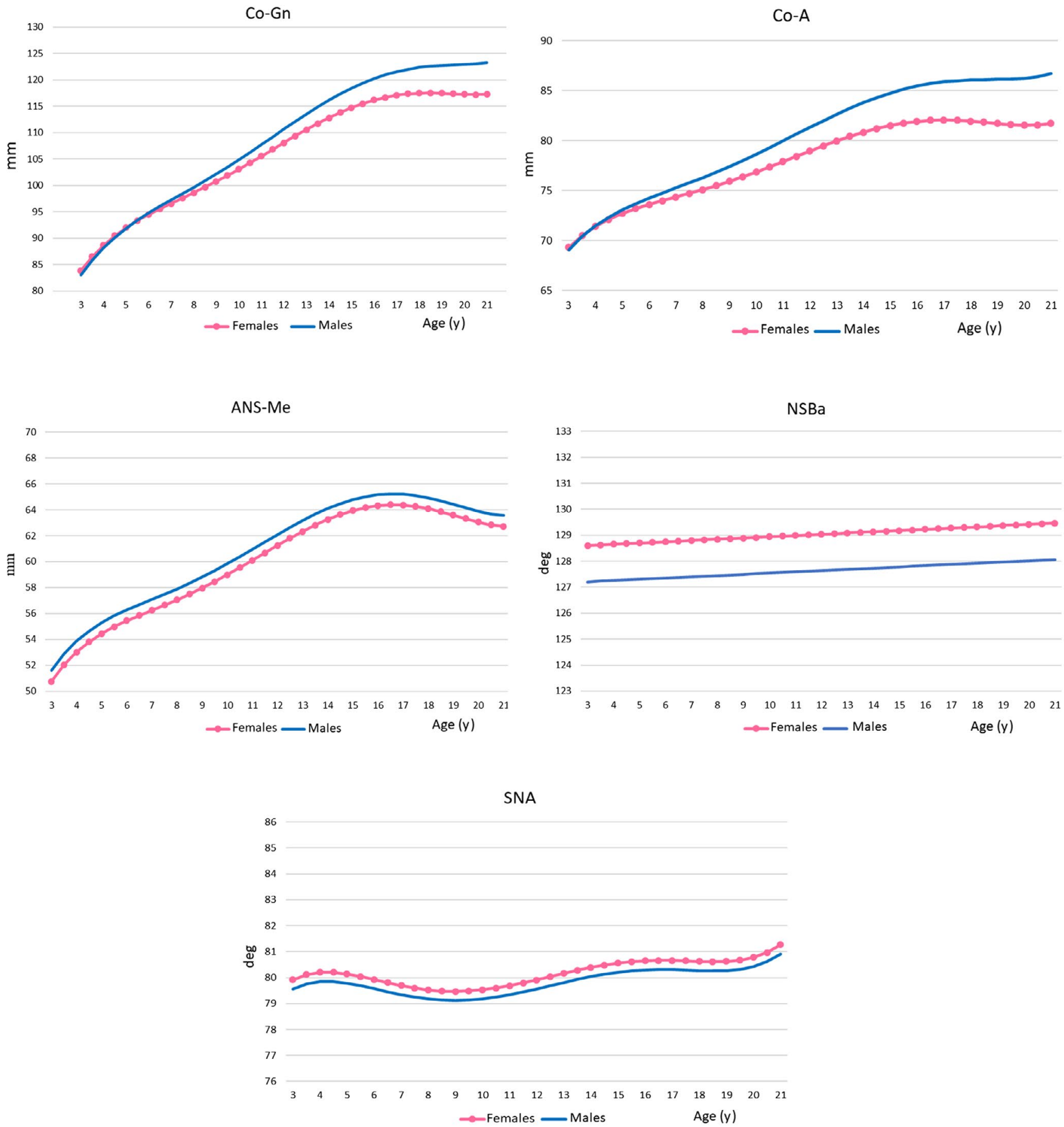

FIGURE 1 Multilevel graphs of the whole sample, for Co-Gn, Co-A, ANS-Me, NSBa and SNA cephalometric variables [Colour figure can be viewed at wileyonlinelibrary.com] 
SNB

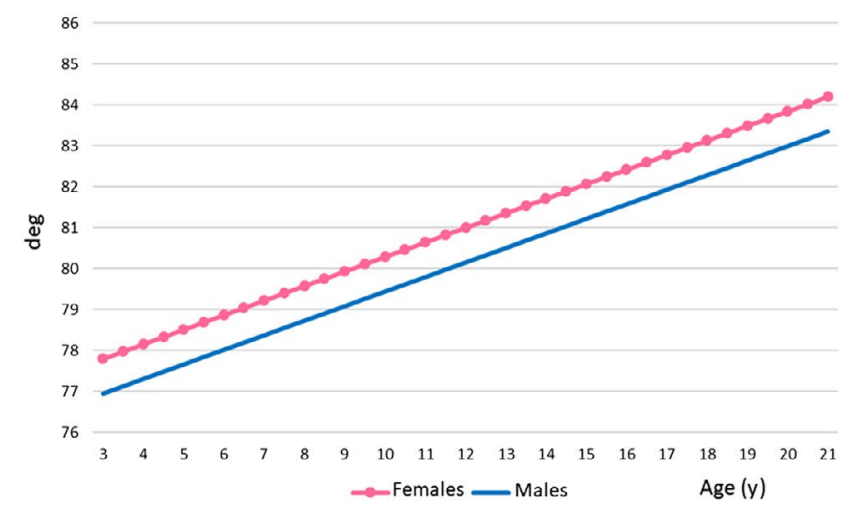

ArGoMe

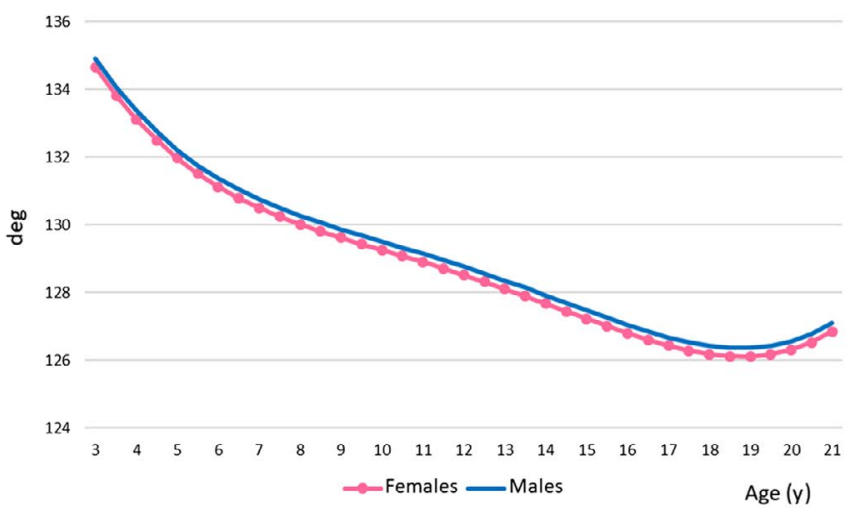

ANB

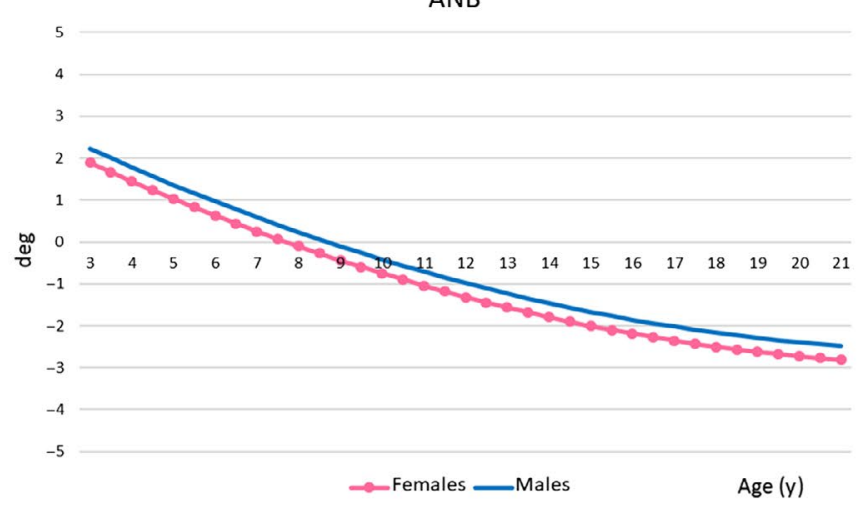

Age (y)
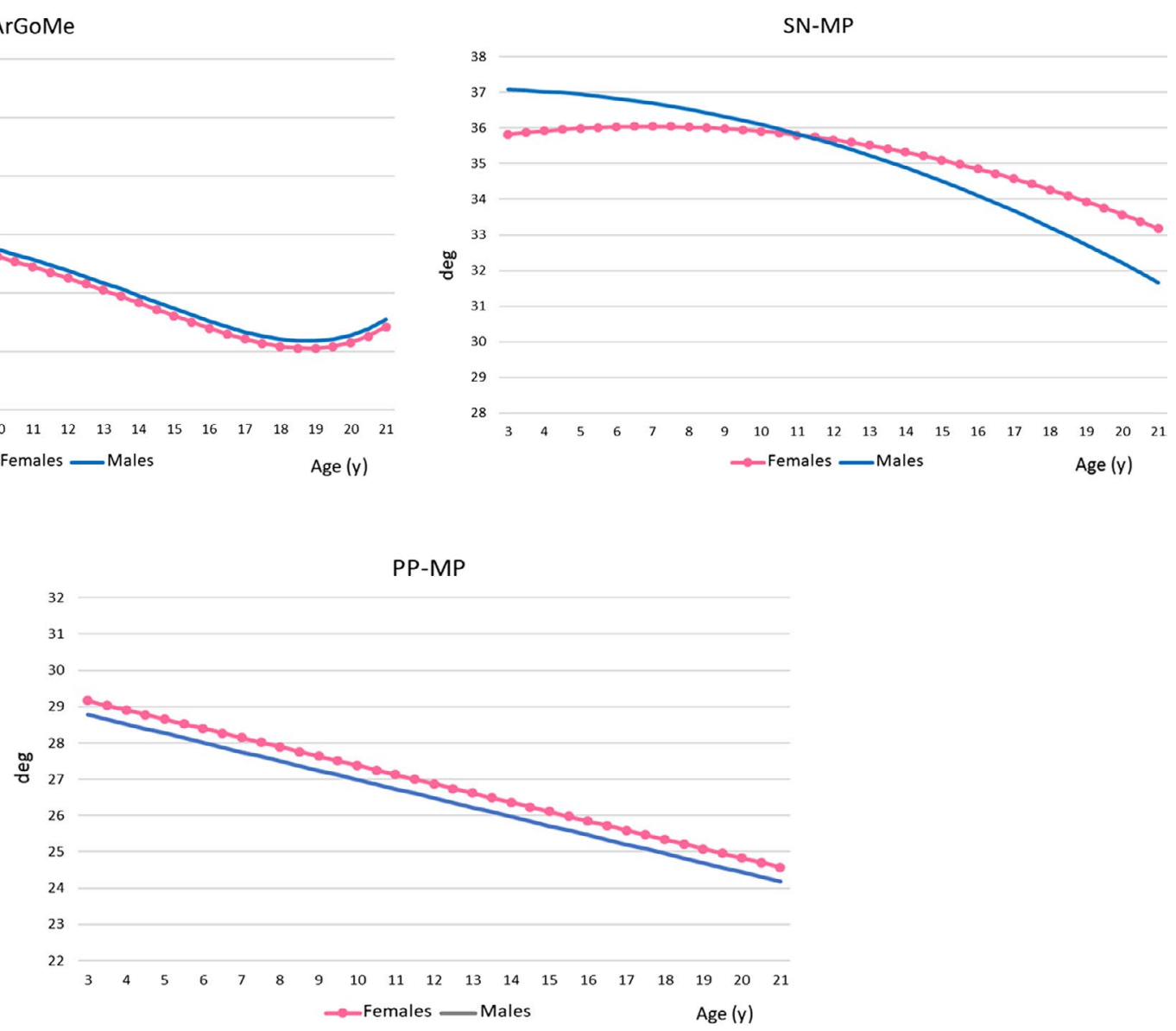

FIGURE 2 Multilevel graphs of the whole sample, for SNB, ANB, ArGoMe, SN-MP and PP-MP cephalometric variables [Colour figure can be viewed at wileyonlinelibrary.com]

the growth estimate occurring the period after 18 years of age should be interpreted cautiously because of the relatively small sample size (16 subjects). Midfacial length growth finished at about 17 years in both males and females. Increases in lower anterior facial height growth also ended at about 17 years in both females and males. There were no gender differences in the observation time, and there were two points of maximum growth at about 3-5 years of age and 11-15 years of age.
The cranial base angle showed no differences in growth between males and females, and it exhibited no appreciable modifications during growth, describing a linear growth model ('Age' was not significant in the model; Table 4). SNA angle showed mild oscillations over time with no differences between males and females.

The increase in the SNB angle was linear and constant over the age range considered, without differences between males and 
females. The ANB angle decreased over time, initially with a more marked tendency (from around 3 to 11 years of age) and then increasingly less accentuated until it stabilized at about 20 years of age at a negative value of about -2.5 degrees. The decrease in the gonial angle was curvilinear with two accentuated decreases at about 3-5 years and 12-16 years. Decrements in the gonial angle ended at about 18 years of age.

The growth curve of the inclination of the mandibular plane in relation to the anterior cranial base was curvilinear in both genders, and it was steeper starting from about 11-13 years. There was interaction between gender and age: males had higher values between approximately 3-7 years, with smaller values after 14 years, compared with females. Females showed a less steep decrease in the growth curve over time.

The growth curve of the inclination of the palatal plane in relation to the mandible plane decreased linearly over time (of about 5 degrees overall). There was much interindividual variability in the quantity of growth for eight out of ten variables (see Figure S1 for Co-Gn variable).

\section{4 | DISCUSSION}

In the present study, a two-level multilevel analysis was able to delineate growth curves for some cephalometric variables over time. The results of this study largely agree with previous longitudinal studies $^{4-6}$ : in fact, a worsening and a lack of spontaneous improvement over time of the skeletal characteristics of Class III malocclusion were described. This lack was particularly evident through a worsening of the ANB angle (associated with a constant increase in the SNB angle), a reduction of the Wits appraisal and an excessive increase in total mandibular length.

The scarcity of data on the untreated Class III subjects made it necessary to undertake this retrospective study that described craniofacial growth in subjects with untreated Class III malocclusion, using cephalometric data elaborated with a curvilinear multilevel model. Multilevel modelling was originally established to analyse clustered data in which measurements were not independent, such as patients treated by the same clinician or clinicians working in the same clinic. ${ }^{31,33}$ Repeated observations can be considered as clustered data with multiple measurements of the same variable made in the same subject, such as repeated cephalometric measurements on the same patient. ${ }^{27,31}$

To evaluate better the growth curve of each variable in each patient, a polynomial curvilinear analysis model was applied to highlight growth variations over time, both in males and females. In fact, the multilevel model can evaluate different growth patterns with the additional variable of 'Gender', resulting in a more complex model. ${ }^{27,31}$ Moreover, random effects were used also to consent the change in growth velocity to vary across children. ${ }^{31}$ Because this type of occlusion invites early intervention and because of the limited prevalence of this malocclusion, data on untreated Class III subjects are increasingly difficult to collect.
In this study, gender differences in the growth curves generated for Co-Gn, Co-A and SN-MP were observed. Total mandibular length (Co-Gn) demonstrated an increase in growth over time, with an average growth of $8.4 \mathrm{~mm}$ between $\mathrm{T} 1$ and T2 (T2-T1 $\mathrm{min}=0.8$ years. $\mathrm{T} 2-\mathrm{T} 1 \mathrm{max}=11.9$ years. $\mathrm{T} 2-\mathrm{T} 1$ mean $=3.8$ years, $\mathrm{SD}=2.7$ years. Table S2).

Growth along Co-Gn continued beyond 15-16 years of age, ending at 17 years of age in females. In males, mandibular growth as measured from Co to $\mathrm{Gn}$ continued until the end of the observation time (21 years and 7 months). As emphasized already, this result should be interpreted with caution due to the limited number of male subjects after the age of 18 years. The persistence of active mandibular growth, well beyond the circumpubertal phase, had been observed only by previous medium-term cross-sectional studies, ${ }^{4,23}$ mostly in males. Previous longitudinal studies revealed that mandibular length had a more pronounced growth in males. ${ }^{4-6}$ In the previous multilevel study $^{6}$ on growth of untreated Class III malocclusion, mandibular length continued to increase rapidly over time, with a greater amount of growth observed in males, as confirmed by the present study.

Midfacial length (Co-A) evidenced an amount of growth at the end of the observation time which was about one third $(3.4 \mathrm{~mm}, \mathrm{Cl}$ : $2.8-3.9 \mathrm{~mm}$ ) of that shown by total mandibular length $(8.4 \mathrm{~mm} . \mathrm{Cl}$ : 7.3- $9.5 \mathrm{~mm}$ ). Maxillary growth deficiency has been confirmed in previous cross-sectional and longitudinal studies ${ }^{4,23}$ and in studies in which small groups of untreated Class III subjects, used as control groups, were followed longitudinally. ${ }^{34,35}$ Previous studies ${ }^{4,23}$ also confirmed gender differences for the Co-A variable. In the study by Wolfe et al, ${ }^{6}$ midfacial length was significantly larger in males than in females, and there were no differences in growth of midfacial length of Class III subjects compared with the control group of Class I subjects. In addition, it was not possible to compare the multilevel curve of growth of the Co-A variable because it had not been reported in previous studies. $^{6}$

Compared with prior cross-sectional studies ${ }^{4}$ and semi-longitudinal studies, ${ }^{5,6}$ in which there was a shorter lower anterior facial height (ANS-Me) in female subjects, the present study did not find gender differences for ANS-Me both in the growth trend and the quantity of growth. A significant increase in this variable during the entire observation interval was detected. This finding is in accordance with those of previous longitudinal studies. ${ }^{5,6}$ In the study by Wolfe and co-workers, ${ }^{6}$ the ANS-Me variable expressed a linear growth increase over time, which, therefore, determined an opening of the mandibular plane angle.

The cranial base angle showed no appreciable changes during growth, both in males and females. The same finding was reached in other longitudinal studies, ${ }^{5,6}$ or a slight closure of this angle was described, albeit not significant. ${ }^{4}$ In the previous multilevel study on this topic ${ }^{6}$ during the observation time (6-16 years of age), there were no significant growth differences between males and females and between Class III subjects and Class I individuals.

The position of the maxilla relative to the cranial base (SNA angle) presented slight but non-significant changes in angulation over time. This outcome suggests that the maxilla was not retruded 
TAB LE 4 Table for the multilevel model of 10 cephalometric variables

\begin{tabular}{|c|c|c|c|c|c|c|}
\hline Term & \multicolumn{3}{|l|}{ Co-Gn } & \multicolumn{3}{|l|}{ Co-A } \\
\hline \multicolumn{7}{|l|}{ Fixed effects } \\
\hline Intercept & 48.948 & 7.194 & & 51.746 & 4.831 & \\
\hline Age & 2.036 & 1.397 & $<.0001$ & 9.793 & 2.413 & $<.0001$ \\
\hline Gender (f) & 2.036 & 1.397 & .1451 & 1.121 & 1.104 & .3099 \\
\hline $\mathrm{Age}^{2}$ & -3.140 & 0.690 & $<.0001$ & -1.785 & 0.463 & $<.0001$ \\
\hline $\mathrm{Age}^{3}$ & 0.2883 & 0.0624 & $<.0001$ & 0.170 & 0.042 & $<.0001$ \\
\hline $\mathrm{Age}^{4}$ & -0.0122 & 0.00268 & $<.0001$ & -0.0746 & 0.00181 & $<.0001$ \\
\hline $\mathrm{Age}^{5}$ & 0.000191 & 0.0000437 & $<.0001$ & 0.000121 & 0.0000296 & $<.0001$ \\
\hline Covariance (Intercept, Age) & -1.357 & 0.524 & & -0.882 & 0.283 & \\
\hline Rx level & 1.425 & 0.148 & & 0.628 & 0.066 & \\
\hline \multirow[t]{2}{*}{-2loglikelihood } & \multicolumn{3}{|l|}{2066.393} & \multicolumn{3}{|l|}{1765.865} \\
\hline & \multicolumn{3}{|l|}{ SNB } & \multicolumn{3}{|l|}{ ANB } \\
\hline Term & Estimated & Standard error & $P$-value & Estimated & Standard error & $P$-value \\
\hline \multicolumn{7}{|l|}{ Fixed effects } \\
\hline Intercept & 75.887 & 0.599 & & 3.671 & 0.533 & \\
\hline Age & 0.355 & 0.033 & $<.0001$ & -0.514 & 0.080 & $<.0001$ \\
\hline \multicolumn{7}{|l|}{ Age $\times$ Gender } \\
\hline \multicolumn{7}{|l|}{$\mathrm{Age}^{3}$} \\
\hline \multicolumn{7}{|l|}{ Random effects (Variance) } \\
\hline Intercept & 20.602 & 3.520 & & 4.605 & 0.578 & \\
\hline Age & 0.080 & 0.017 & $<.0001$ & & & \\
\hline Covariance (Intercept, Age) & -0.721 & 0.211 & & & & \\
\hline Rx level & 0.400 & 0.043 & & 0.746 & 0.063 & \\
\hline -2loglikelihood & 1604.539 & & & 1487.465 & & \\
\hline
\end{tabular}

relative to the cranial base, with respect to normal values. ${ }^{36}$ At the same time, the forward growth of the maxilla was deficient compared with the excessive mandibular growth in a forward and downward direction. Moreover, the multilevel growth curve for the SNA variable was not present. Even in that study, ${ }^{6}$ the position of the maxilla (evaluated by the SNA angle and Co-A) was not retruded at the end of growth, despite the end of the time evaluated was only 16 years of age.

The position of the mandible relative to the cranial base, measured with the SNB angle, progressed over time without gender differences. Predictably, the increase in SNB angle in the untreated Class III malocclusion was more pronounced than normal, ${ }^{36}$ with a total increase of about 6 degrees, on average, during the observation time, both for males and females.

Such a forward growth of the mandible, demonstrated by the increase in the SNB angle, has been described by previous studies on craniofacial growth of untreated Class III malocclusion. Baccetti et $\mathrm{al}^{4}$ conducted a longitudinal study in which the increase in the SNB angle was more than double of that of the SNA angle. Wolfe et al, ${ }^{6}$ regarding the SNB angle, reported a statistically significant increase over time, without differences between males and females. The gonial angle showed a reduction in angulation over time.

The same growth trend was demonstrated in the multilevel graphics in the study of Wolfe et al, ${ }^{6}$ but also in the study of Zionic 


\begin{tabular}{|c|c|c|c|c|c|c|c|c|}
\hline \multicolumn{3}{|l|}{ ANS-Me } & \multicolumn{3}{|l|}{ NSBa } & \multicolumn{3}{|l|}{ SNA } \\
\hline Estimated & Standard error & $P$-value & Estimated & Standard error & $P$-value & Estimated & Standard error & $P$-value \\
\hline 33.857 & 5.214 & & 127.064 & 0.859 & & 76.603 & 4.319 & \\
\hline 10.194 & 2.613 & $<.0001$ & 0.0478 & 0.0431 & .2674 & 4.061 & 2.171 & .0613 \\
\hline-1.901 & 0.500 & .0001 & & & & -0.936 & 0.417 & .0248 \\
\hline 0.180 & 0.045 & $<.0001$ & & & & 0.0934 & 0.0378 & .0135 \\
\hline-0.0784 & 0.00194 & $<.0001$ & & & & -0.00417 & 0.00162 & .0100 \\
\hline 0.000125 & 0.0000317 & $<.0001$ & & & & 0.0000689 & 0.0000265 & .0093 \\
\hline-0.793 & 0.300 & & -1.485 & 0.414 & & -0.473 & 0.184 & \\
\hline 0.716 & 0.075 & & 0.960 & 0.099 & & 0.545 & 0.0555 & \\
\hline 1816.237 & & & 1904.475 & & & 1646.329 & & \\
\hline \multicolumn{2}{|l|}{ ArGoMe } & \multirow[b]{2}{*}{$P$-value } & \multicolumn{2}{|l|}{ SN-MP } & \multirow[b]{2}{*}{$P$-value } & \multicolumn{3}{|l|}{ PP-MP } \\
\hline Estimated & Standard error & & Estimated & Standard error & & Estimated & Standard error & $P$-value \\
\hline 143.133 & 3.925 & & 37.071 & 0.999 & & 29.540 & 0.767 & \\
\hline-3.905 & 1.492 & .0089 & 0.0473 & 0.1098 & .6666 & -0.255 & 0.043 & $<.0001$ \\
\hline 28.586 & 6.447 & & 35.218 & 4.209 & & 24.208 & 4.918 & \\
\hline 0.113 & 0.035 & .0009 & & & & 0.110 & 0.027 & $<.0001$ \\
\hline-0.464 & 0.395 & & & & & -0.327 & 0.295 & \\
\hline 1.956 & 0.194 & & 1.364 & 0.116 & & 1.089 & 0.110 & \\
\hline 2092.397 & & & 1941.825 & & & 1939.348 & & \\
\hline
\end{tabular}

Alexander et $\mathrm{al}^{5}$ in which the increments in growth at the 6-16 age intervals were almost always negative (for 16 age intervals out of 21). Similarly, Baccetti et al ${ }^{4}$ described a negative T2-T1 difference of the Ar-Go-Me angle (-2.4 degrees).

The inclination of the mandibular plane in relation to the anterior cranial base (SN-MP) and the inclination of the palatal plane in relation to the mandible plane (PP-MP) were not taken into account in previous longitudinal studies. ${ }^{4,5}$ In these investigations, however, the angulation of vertical values ( $\mathrm{FH}$ to occlusal plane, $\mathrm{FH}$ to palatal plane and MPA angle) decreased over time, as reported in the current study. Regarding the study of Wolfe et al, $^{6}$ these two variables (SN-MP, PP-MP) were not considered in the multilevel analysis. The mandibular plane angle, however, was significantly larger in the Class III group at 11 years of age, and the growth curve of this variable decreased lightly over time.

Interindividual variability was statistically significant for eight (Co-Gn, Co-A, ANS-Me, SNA, SNB, ArGoMe, PP-MP, NSBa) out of ten variables considered. Variability can be due to a summation of minor effects from a variety of different genes and/or from the influence of epigenetic factors. ${ }^{37-39}$

A limit of this study is that only $28 / 144$ subjects have more than three observations, and 88/144 subjects have only two observations. These results reduce the capacity to correctly understand the longitudinal variation of cephalometric measures within subject. 
Another limit of this study was that the reliability of reported outcomes below 6-7 years and above 18 years was reduced due to the few longitudinal observations available.

\section{5 | CONCLUSIONS}

In summary, it emerged that:

- A growth spike for Co-Gn, Co-A and ANS-Me and increase in the angulation for SNA at 11-15 years of age were found.

- Untreated Class III malocclusion progressively worsened over time and did not show spontaneous improvement.

- Gender differences were found for the Co-Gn, Co-A and SN-MP variables. The spike in growth at 11-15 years was delayed in males by about 1 year in comparison with females, both for the total mandibular length and for midfacial length. In addition, this spike in growth during the circumpubertal growth period continued for about a year longer in males, and there was a greater increase in length of about $5 \mathrm{~mm}$ in males compared with females.

- Class III malocclusion had a protruded and larger mandible, while the maxilla was not retruded at the end of growth.

- Significant mandibular growth continued for a long time after the pubertal phase in untreated Class III subjects. In particular, growth in mandibular length (Co-Gn) ended about 17 years of age for females while in males mandibular growth continued after 18 years. Growth in midfacial length finished at about 17 years in both females and males. Growth in lower anterior facial height ended at about 17 years in both genders.

\section{CONFLICT OF INTEREST}

The authors declared no potential conflicts of interest with respect to the research, authorship and/or publication of this article.

\section{ORCID}

Lorenzo Franchi iD https://orcid.org/0000-0002-2072-460X

\section{REFERENCES}

1. Pavoni C, Mucedero M, Baccetti T, Franchi L, Polimeni A, Cozza P. The effects of facial mask/bite block therapy with or without rapid palatal expansion. Prog Orthod. 2009;10(1):20-28.

2. Franchi L, Pavoni C, Cerroni S, Cozza P. Thin-plate spline analysis of mandibular morphological changes induced by early class III treatment: a long-term evaluation. Eur J Orthod. 2014;36(4):425-430.

3. Rongo R, D'Anto V, Bucci R, Polito I, Martina R, Michelotti A. Skeletal and dental effects of Class III orthopaedic treatment: a systematic review and meta-analysis. J Oral Rehabil. 2017;44(7):545-562.

4. Baccetti T, Franchi L, McNamara JA Jr. Growth in the untreated Class III subject. Semin Orthod. 2007;13(3):130-142.

5. Alexander AE, McNamara JA Jr, Franchi L, Baccetti T. Semilongitudinal cephalometric study of craniofacial growth in untreated Class III malocclusion. Am J Orthod Dentofacial Orthop. 2009;135(6):700.e1-714.e14.

6. Wolfe SM, Araujo E, Behrents RG, Buschang PH. Craniofacial growth of Class III subjects six to sixteen years of age. Angle Orthod. 2011;81(2):211-216.
7. Auconi P, Scazzocchio M, Caldarelli G, Nieri M, McNamara JA Jr, Franchi L. Understanding interactions among cephalometrics variables during growth in untreated Class III subjects. Eur J Orthod. 2017;39(4):395-401.

8. Barelli E, Ottaviani E, Auconi P, et al. Exploiting the interplay between cross-sectional and longitudinal data in Class III malocclusion patients. Sci Rep. 2019;9(1):6189.

9. Hardy D, Cubas Y, Orellana M. Prevalence of angle class III malocclusion: a systematic review and meta-analysis. Open J Epidemiol. 2012;2:75-82.

10. Huber RE, Reynolds JW. A dentofacial study of male students at the University of Michigan in the physical hardening program. Am J Orthod Oral Surg. 1946;32:1-21.

11. Mills LF. Epidemiologic studies of occlusion. IV. The prevalence of malocclusion in a population of 1,455 school children. J Dent Res. 1966;45(2):332-336.

12. Sidlauskas $A$, Lopatiene $K$. The prevalence of malocclusion among 7-15-year-old Lithuanian schoolchildren. Medicina (Kaunas). 2009;45(2):147-152.

13. Ferro R, Besostri A, Olivieri A, Stellini E. Prevalence of occlusal traits and orthodontic treatment need in 14 year-old adolescents in Northeast Italy. Eur J Paediatr Dent. 2016;17(1):36-42.

14. Lew KK, Foong WC, Loh E. Malocclusion prevalence in an ethnic Chinese population. Aust Dent J. 1993;38(6):442-449.

15. Soh J, Sandham A, Chan YH. Occlusal status in Asian male adults: prevalence and ethnic variation. Angle Orthod. 2005;75(5):814-820.

16. Shen L, He F, Zhang C, Jiang H, Wang J. Prevalence of malocclusion in primary dentition in mainland China, 1988-2017: a systematic review and meta-analysis. Sci Rep. 2018;8(1):4716.

17. Doraczynska-Kowalik A, Nelke $K H$, Pawlak W, Sasiadek MM, Gerber H. Genetic factors involved in mandibular prognathism. J Craniofac Surg. 2017;28(5):e422-e431.

18. Liu H, Wu C, Lin J, Shao J, Chen Q, Luo E. Genetic etiology in nonsyndromic mandibular prognathism. J Craniofac Surg. 2017;28(1):161-169.

19. Guyer EC, Ellis EE, McNamara JA Jr, Behrents RG. Components of class III malocclusion in juveniles and adolescents. Angle Orthod. 1986;56(1):7-30.

20. Tollaro I, Baccetti T, Bassarelli V, Franchi L. Class III malocclusion in the deciduous dentition: a morphological and correlation study. Eur J Orthod. 1994;16(5):401-408.

21. Battagel JM. The aetiological factors in Class III malocclusion. Eur J Orthod. 1993;15(5):347-370.

22. Baccetti T, Reyes BC, McNamara JA Jr. Gender differences in Class III malocclusion. Angle Orthod. 2005;75(4):510-520.

23. Reyes BC, Baccetti T, McNamara JA Jr. An estimate of craniofacial growth in Class III malocclusion. Angle Orthod. 2006;76(4):577-584.

24. Kuc-Michalska M, Baccetti T. Duration of the pubertal peak in skeletal Class I and Class III subjects. Angle Orthod. 2010;80(1):54-57.

25. Arboleda C, Buschang PH, Camacho JA, Botero P, Roldan S. A mixed longitudinal anthropometric study of craniofacial growth of Colombian mestizos 6-17 years of age. Eur J Orthod. 2011;33(4):441-449.

26. Jacob HB, Buschang $\mathrm{PH}$. Vertical craniofacial growth changes in French-Canadians between 10 and 15 years of age. Am J Orthod Dentofacial Orthop. 2011;139(6):797-805.

27. Tu YK, Chiu YW, Pandis N. Analyzing longitudinal orthodontic data. Part 1: multilevel linear and curvilinear models. Am J Orthod Dentofacial Orthop. 2013;144(3):481-486.

28. Nahhas RW, Valiathan M, Sherwood RJ. Variation in timing, duration, intensity, and direction of adolescent growth in the mandible, maxilla, and cranial base: the Fels longitudinal study. Anat Rec (Hoboken). 2014;297(7):1195-1207.

29. von Elm E, Altman DG, Egger $M$, et al. The Strengthening the Reporting of Observational Studies in Epidemiology (STROBE) 
statement: guidelines for reporting observational studies. J Clin Epidemiol. 2008;61(4):344-349.

30. Levin AS, McNamara JA Jr, Franchi L, Baccetti T, Frankel C. Short-term and long-term treatment outcomes with the FR-3 appliance of Frankel. Am J Orthod Dentofacial Orthop. 2008;134(4):513-524.

31. Tu YK. Analyzing longitudinal orthodontic data. In: Pandis N, ed. Biostatistics in Orthodontics. Bern, Switzerland: Zahnmedizinische Kliniken der Universität Bern; 2014:113-133.

32. Hoeksma JB, van der Beek MC. Multilevel modelling of longitudinal cephalometric data explained for orthodontists. Eur J Orthod. 1991;13(3):197-201.

33. Goldstein H. Multilevel Statistical Models, 4th ed. West Sussex, UK: John Wiley \& Sons; 2011:358.

34. Macdonald KE, Kapust AJ, Turley PK. Cephalometric changes after the correction of class III malocclusion with maxillary expansion/facemask therapy. Am J Orthod Dentofacial Orthop. 1999;116(1):13-24.

35. Westwood PV, McNamara JA Jr, Baccetti T, Franchi L, Sarver DM. Long-term effects of Class III treatment with rapid maxillary expansion and facemask therapy followed by fixed appliances. Am J Orthod Dentofacial Orthop. 2003;123(3):306-320.

36. Bhatia SN, Leighton BC. A Manual of Facial Growth: A Computer Analysis of Longitudinal Cephalometric Growth Data, 1st ed. Oxford, UK: Oxford University Press; 1993:1-401.
37. Cruz RM, Krieger H, Ferreira R, Mah J, Hartsfield J Jr, Oliveira S. Major gene and multifactorial inheritance of mandibular prognathism. Am J Med Genet A. 2008;146A(1): 71-77.

38. Xue F, Wong RW, Rabie AB. Genes, genetics, and Class III malocclusion. Orthod Craniofac Res. 2010;13(2):69-74.

39. Ko JM, Suh YJ, Hong J, Paeng JY, Baek SH, Kim YH. Segregation analysis of mandibular prognathism in Korean orthognathic surgery patients and their families. Angle Orthod. 2013;83(6):1027-1035.

\section{SUPPORTING INFORMATION}

Additional supporting information may be found online in the Supporting Information section.

How to cite this article: Rutili V, Nieri M, Giuntini V, McNamara JA Jr, Franchi L. A multilevel analysis of craniofacial growth in subjects with untreated Class III malocclusion. Orthod Craniofac Res. 2020;23:181-191. https://doi.org/10.1111/ocr.12356 